\title{
Multi-Quartz Enhanced Photoacoustic Spectroscopy with Different Acoustic Microresonator Configurations
}

\author{
Huadan Zheng, ${ }^{1,2}$ Xukun Yin, ${ }^{1,2}$ Lei Dong, ${ }^{1,2}$ Hongpeng Wu, ${ }^{1,2}$ Xiaoli Liu, \\ Weiguang Ma, ${ }^{1,2}$ Lei Zhang, ${ }^{1,2}$ Wangbao Yin, ${ }^{1,2}$ and Suotang Jia ${ }^{1,2}$ \\ ${ }^{1}$ State Key Laboratory of Quantum Optics and Quantum Optics Devices, Institute of Laser Spectroscopy, Shanxi University, \\ Taiyuan 030006, China \\ ${ }^{2}$ Collaborative Innovation Center of Extreme Optics, Shanxi University, Taiyuan 030006, China
}

Correspondence should be addressed to Lei Dong; donglei@sxu.edu.cn

Received 16 September 2015; Accepted 22 October 2015

Academic Editor: Arnaud Cuisset

Copyright (C) 2015 Huadan Zheng et al. This is an open access article distributed under the Creative Commons Attribution License, which permits unrestricted use, distribution, and reproduction in any medium, provided the original work is properly cited.

\begin{abstract}
Acoustic microresonators were added to the recently developed multi-QTF based QEPAS spectrophone to enhance the signal amplitude. Two kinds of "on-beam" configurations were experimentally investigated in detail. The developed multi-QTF based "on-beam" spectrophone had a signal enhancement of 1.6 times compared with the traditional single QTF based "onbeam" spectrophone, with the approximate noise level. A normalized noise equivalent absorption coefficient $(1 \sigma)$ of $1.24 \times$ $10^{-9} \mathrm{~W} \cdot \mathrm{cm}^{-1} \cdot \mathrm{Hz}^{-1 / 2}$ was obtained for water vapor detection at atmospheric pressure.
\end{abstract}

\section{Introduction}

Laser absorption spectroscopy for trace gas detection has gained considerable interest over the past decades. Among optical spectroscopic techniques, photoacoustic spectroscopy (PAS) is a well-established sensitive approach, with the advantages of zero-background nature, excitation-wavelength independence, and linear dependence of signal amplitudes on excitation powers [1-4]. As a variation of PAS, quartz enhanced photoacoustic spectroscopy (QEPAS), by use of a quartz tuning fork (QTF) instead of a traditional microphone, is a booming technique for trace gas detection that has been widely used for environmental monitoring, industrial process control analysis, combustion processes, and detection of toxic and flammable gases, as well as explosives [5]. The sharply high $Q$ value of the quartz tuning fork (QTF) not only facilitates the accumulation of the acoustic energy generated from the relaxation transition of gas molecules, but also makes the QEPAS based spectrophone immune to environmental noise.

Acoustic microresonators (AmRs) were usually used in QEPAS spectrophone to enhance the acoustic wave oscillation, induced by the modulated beam in the absorbing gas, by virtue of the coupling effects between the QTF and the AmRs [6-8]. Two sophisticated QEPAS spectrophones with AmRs assembled in "on-beam" and "off-beam" configurations have been developed to improve the performance of bare-QTF based QEPAS sensors $[9,10]$. The spectrophone assembled in the "on-beam" configuration [9], with two AmRs positioned closely on each side of the QTF, offers a signal-to-noise ratio (SNR) enhancement factor of $\sim 30$. The spectrophone assembled in the "off-beam" configuration [10], having a single AmR with a small slit made in the middle placed alongside the QTF, can simplify optical alignment and be applied to excitation sources with a low spatial radiation quality. Recently, some novel QEPAS spectrophones were also reported. Double acoustic microresonator quartz-enhanced photoacoustic spectroscopy which employed the spectrophone that consisted of a QTF and two sets of the "onbeam" AmRs was developed for optical signal addition and multigas rapid spectral measurement with a response time of $5 \mathrm{~ms}$ [1]. Multi-quartz enhanced photoacoustic spectroscopy (M-QEPAS) uses a pair of QTFs with similar resonance frequencies connected in parallel, instead of a single QTF in the traditional QEPAS spectrophone, to enhance the signal amplitude through coupling effect between the two QTFs [11]. 


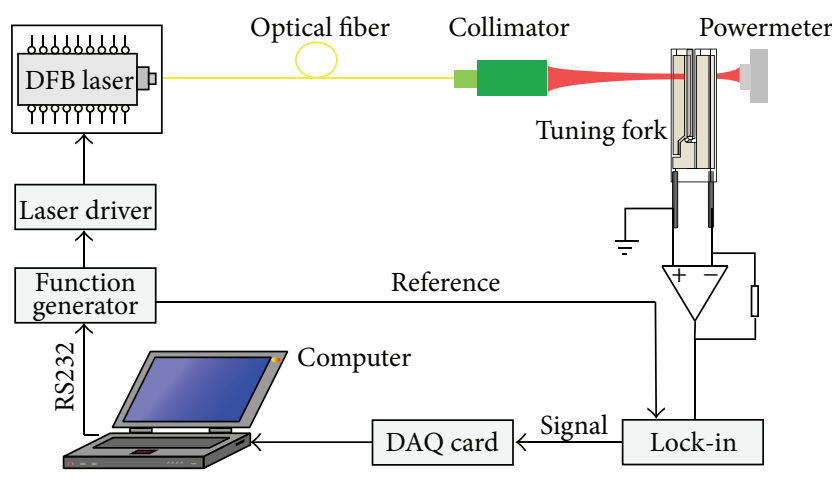

Figure 1: Schematic of the experimental setup.

M-QEPAS realized a 1.7 times signal enhancement for the water vapor detection in the air, compared to the QEPAS sensor with a single bare QTF. But there were no AmRs configured in the M-QEPAS spectrophone.

In this paper, AmRs were added to the M-QEPAS spectrophone to further enhance the signal amplitude of the sensor. Two kinds of multi-QTF based "on-beam" QEPAS spectrophone were proposed. The resonance curves of the proposed QEPAS spectrophones were measured to determine their resonance frequencies and $Q$ values. For its practical implementation, water vapor in the laboratory air was selected as the target analyte to evaluate the sensor performance in detail. The distance between the AmR and the QTF in the multiQTF based "on-beam" QEPAS spectrophone was optimized to obtain the maximum signal amplitude.

\section{Experimental Setup}

The schematic diagram of the experimental setup was depicted in Figure 1. A near-infrared fiber-coupled distributed-feedback (DFB) diode laser was employed as excitation source to generate photoacoustic signals. The output wavelength of the DFB laser was $1368.69 \mathrm{~nm}$, with the temperature and the current of the DFB laser set to $27^{\circ} \mathrm{C}$ and $80 \mathrm{~mA}$. The laser wavelength can be coarsely and finely tuned by scanning the temperature and the current, respectively. A commercial $\sim 32 \mathrm{kHz}$ quartz tuning fork was used as an acoustic transducer. The laser current was modulated at $\sim 16 \mathrm{kHz}$, corresponding to a half of the QTF resonance frequency. The output beam from the DFB laser was focused to pass through the gap between the QTF prongs by means of a fiber coupled focuser. The acoustic wave induced by the modulated laser beam effectively pushed the prongs of the QTF to vibrate. The electrical signals generated by piezoelectric effect of the QTF were processed by a transimpedance preamplifier and then were fed to a lock-in amplifier, demodulating the signal at $2 f$ mode and retrieving the gas concentrations.

The output power and wavelength of the DFB laser as a function of current were plotted in Figure 2. The current of the DFB laser was scanned from $15 \mathrm{~mA}$ to $125 \mathrm{~mA}$ at a constant temperature of $23.95^{\circ} \mathrm{C}$, corresponding to a wavelength range from $7305.8 \mathrm{~cm}^{-1}$ to $7309.9 \mathrm{~cm}^{-1}$. Two $\mathrm{H}_{2} \mathrm{O}$ absorption lines, located at $7306.75 \mathrm{~cm}^{-1}$ and $7308.82 \mathrm{~cm}^{-1}$, were

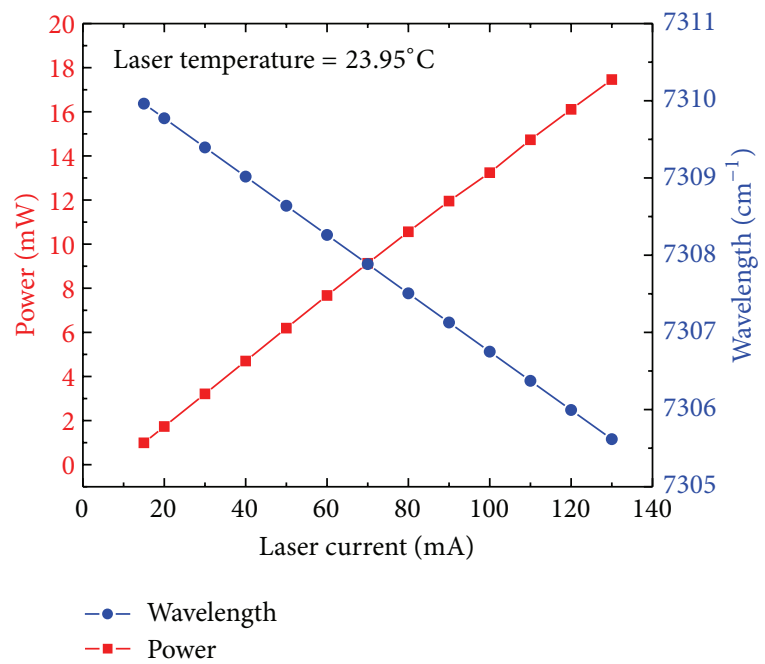

FIGURE 2: Output power and wavelength of the laser as a function of current.

covered within the scanning range. According to the HITRAN database, the two $\mathrm{H}_{2} \mathrm{O}$ absorption lines have the line intensities of $1.8 \times 10^{-20} \mathrm{~cm} / \mathrm{mol}$ and $6.9 \times 10^{-22} \mathrm{~cm} / \mathrm{mol}$, respectively, as shown in Figure 3(a). The relevant $2 f$ signal, obtained from the QEPAS sensor by use of the spectrophone based on a bare QTF, was shown in Figure 3(b). A lower detection limit can be achieved by selecting a stronger target line, but to avoid the overload of the lock-in amplifier, the weaker line at $7308.82 \mathrm{~cm}^{-1}$ was selected as the target line in this experiment.

Figure 4 shows four different QEPAS spectrophones: (a) single bare QTF based spectrophone, (b) traditional single QTF based "on-beam" spectrophone, and (c), (d) multi-QTF based "on-beam" spectrophones. In Figure 4(c), two QTFs were placed in a line with a gap of $20 \mu \mathrm{m}$, and two AmRs were positioned closely on the one side of each QTFs with the same gap of $20 \mu \mathrm{m}$. The distance from the top of the QTF prongs to the center of the AmRs was $0.7 \mathrm{~mm}$. In Figure 4(d), an additional QTF was added to a traditional single QTF based "on-beam" spectrophone. The distance from the top of the QTF prongs to the center of the AmRs and the gaps between the QTF \#3 and the AmRs were the same as in Figure 4(c). This configuration is equivalent to two traditional half "onbeam" spectrophones connected in series [12]. The geometric parameters of all the AmRs used in Figures 4(b)-4(d) are the same as in [13].

\section{Results and Discussion}

A function generator was used to generate sinusoidal waves to excite the QTF, and the output signals of the QTF at different excitation frequencies were recorded as the resonance curves. The resonance frequency $f_{0}$ and $Q$ value of the QTF can be obtained from Lorentz fitting of the square of the resonance curve [14]. The $Q$ value is defined as $f_{0} / \Delta f$, where $\Delta f$ represents the full width at half maximum (FWHM) of the resonance curve. The resonance curve of the single bare 


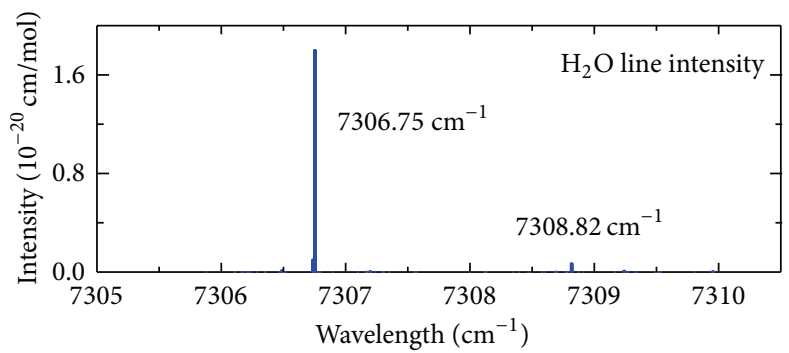

(a)

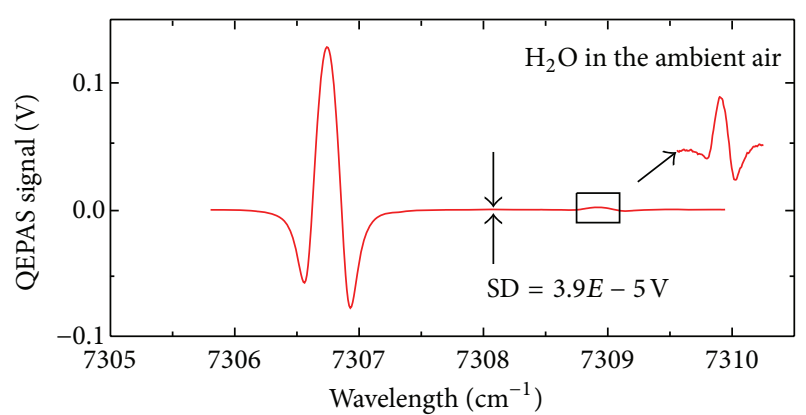

(b)

FIgURE 3: (a) Locations and intensities of $\mathrm{H}_{2} \mathrm{O}$ absorption lines from the HITRAN database; (b) $2 f$ signal obtained from the QEPAS sensor by use of a single bare QTF based spectrophone; SD: standard deviation.

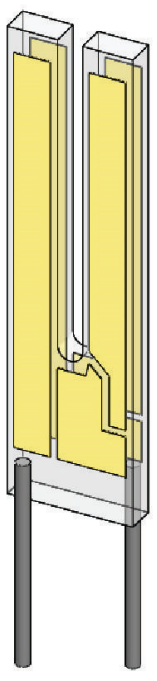

(a)

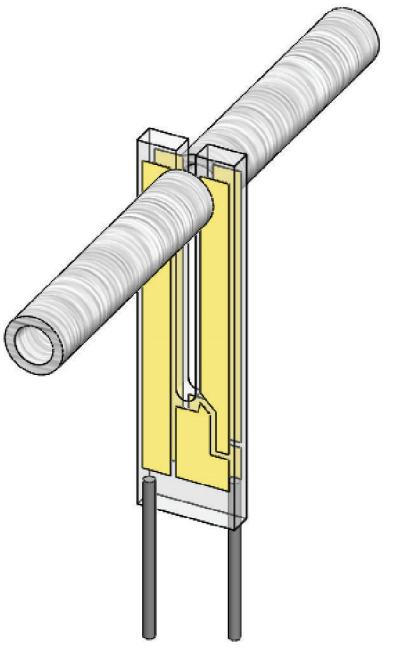

(b)

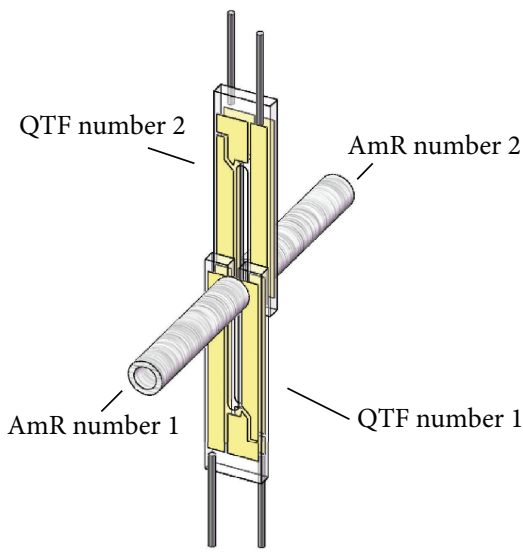

(c)

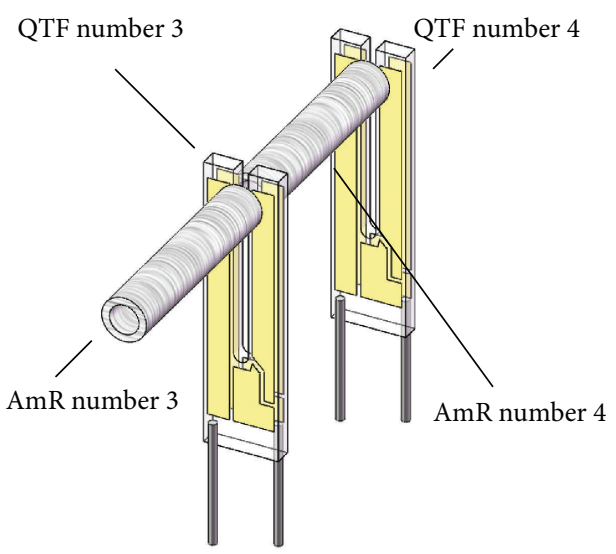

(d)

Figure 4: (a) Single bare QTF based spectrophone, (b) single QTF based “on-beam" spectrophone, and (c), (d) multi-QTF based "on-beam" spectrophones. The geometric parameters of all the AmRs used in Figure 4 are $4.4 \mathrm{~mm}$ in length and $0.6 \mathrm{~mm}$ in inner diameters, respectively.

QTF based spectrophone (Figure 4(a)) and the traditional single QTF based “on-beam" spectrophone (Figure 4(b)) were shown in Figure 5. The frequency of the sinusoidal wave from the function generator was scanned from $32720 \mathrm{~Hz}$ to $32780 \mathrm{~Hz}$ with peak to peak amplitude of $317 \mathrm{mV}$. A standard
QTF has the resonance frequency of $32768 \mathrm{~Hz}$ and $Q$ value of $80,000-100,000$ in a sealed vacuum metallic package. The frequency and the $Q$ value decreased to $32748.8 \mathrm{~Hz}$ and 8396 in the air, after removal of the metallic housing. With the traditional single QTF based "on-beam" QEPAS 


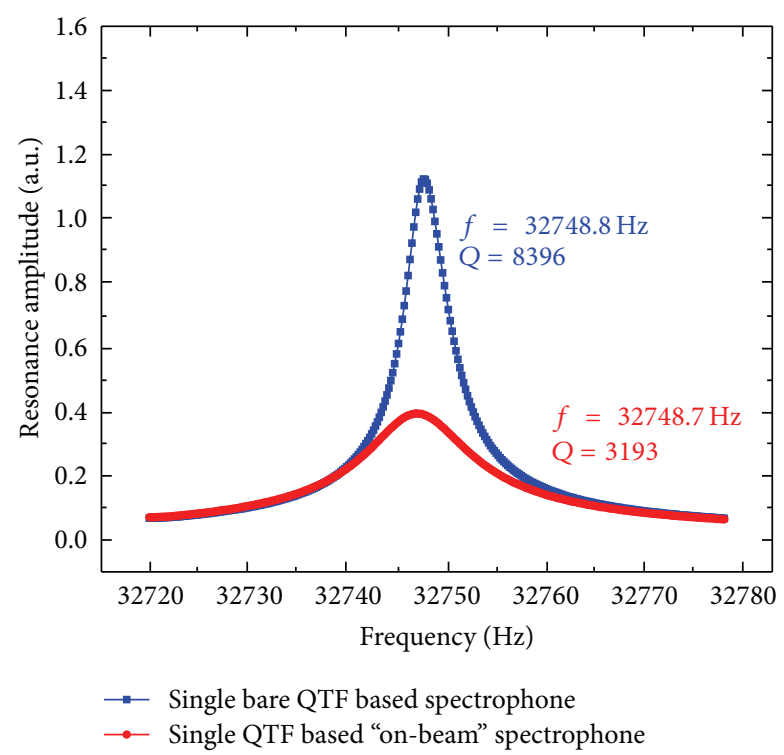

FIGURE 5: Resonance curves of a single bare QTF based spectrophone and a traditional single QTF based "on-beam" spectrophone measured at atmospheric pressure in the air.

TABLE 1: Parameters of multi-QTF based "on-beam" spectrophone (Figure 4(c)).

\begin{tabular}{lccc}
\hline & QTF \#1 & QTF \#2 & Multi-QTF \\
\hline Resonance frequency (Hz) & 32747.29 & 32744.15 & 32746.93 \\
Q value & 5660 & 2923 & 6918 \\
\hline
\end{tabular}

spectrophone, the $Q$ factor further decreased to 3193 , due to the strong coupling effect between the QTF and AmRs. The lower $Q$ value means more acoustic energy exchange and storage between the QTF and the AmRs.

The performance of the multi-QTF based "on-beam" spectrophone shown in Figure 4(c) was first evaluated. The resonance curves of both QTFs were measured independently, and the corresponding parameters were listed in Table 1. QTFs \#1 and \#2 showed the approximate frequency. As a result, only one peak value on the response curve was observed, after connecting the electrodes of the two QTFs in parallel. The Q value of 5660 and 2923 for each QTF indicated that both the QTFs were coupled with the AmRs independently. Due to the strong coupling effect between the multi-QTF and the AmRs, the multi-QTF based "on-beam" spectrophone, shown in Figure 4(c), exhibited a $Q$ value of 6918. Figure 6 shows the resonance curves obtained for QTF \#1, QTF \#2, and multi-QTF using the configuration of Figure 4(c), indicating that when two QTFs were connected in parallel and strongly coupled with the AmRs, Q-factor of multi-QTFs can be higher than each of the two QTFs.

The modulation depth of the laser was optimized with the bare QTF based spectrophone. The laboratory air containing $1.26 \% \mathrm{H}_{2} \mathrm{O}$ was selected as the target analyte. The signal amplitudes and spectral profiles of the QEPAS $2 f$ signal as a function of laser modulation depth were plotted in Figures 7(a) and 7(b). As shown in Figures 7(a) and 7(b), with

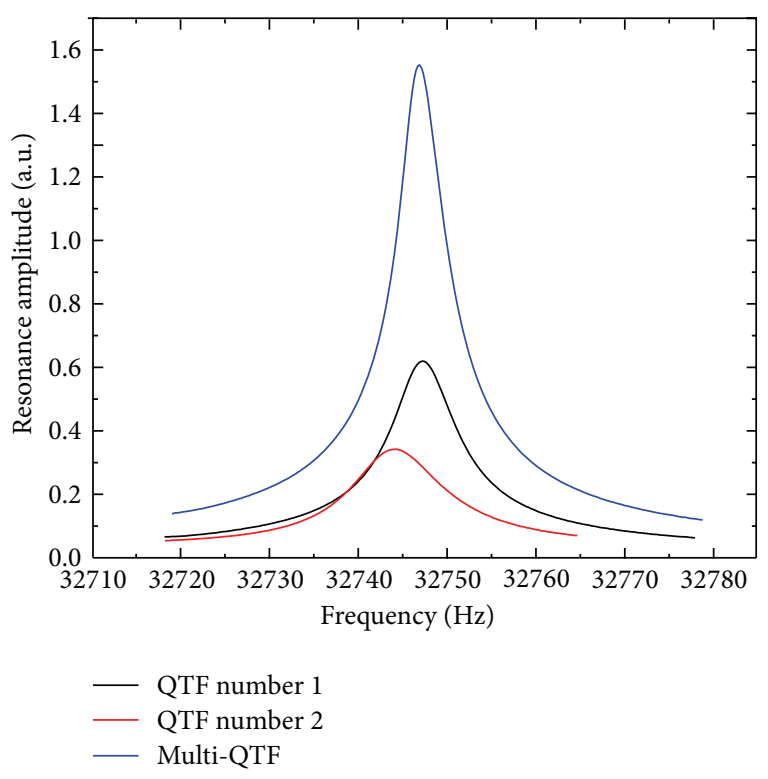

FIGURE 6: Resonance curves obtained for QTF \#1, QTF \#2, and Multi-QTF using the configuration of Figure 4(c).

TABLE 2: Parameters of multi-QTF based “on-beam” spectrophone (Figure 4(d)).

\begin{tabular}{lccc}
\hline & QTF \#3 & QTF \#4 & Multi-QTF \\
\hline Resonance frequency (Hz) & 32744.93 & 32746.96 & 32745.68 \\
Q value & 5384 & 5540 & 5714 \\
\hline
\end{tabular}

the modulation depth increasing from 5 to $20 \mathrm{~mA}$, both the amplitude and spectra width of the QEPAS signal increased monotonously. However, with the modulation depth increasing from 13 to $20 \mathrm{~mA}$, the signal amplitude increased only 9.4\% (from $1.71 \mathrm{mV}$ to $1.96 \mathrm{mV}$ ), shown in inset (j), but the spectra width increased $43.6 \%$ (from $0.44 \mathrm{~cm}^{-1}$ to $0.63 \mathrm{~cm}^{-1}$ ), shown in inset (i). Therefore, an optimum modulation depth of $13 \mathrm{~mA}$ was selected, considering the spectral broadening.

QTFs \#1 and \#2 were first evaluated independently. The obtained signals were demonstrated in Figure 8(a). QTFs \#1 and \#2 showed the signal amplitudes of $41.5 \mathrm{mV}$ and $37.5 \mathrm{mV}$ which are 23.3 times and 21 times higher than that of the single bare QTF based spectrophone. This indicates that both QTFs \#1 and \#2 were strongly coupled with the AmRs assembled in the "on-beam" configuration. The QEPAS signal, obtained with the two QTFs' electrodes connected in parallel, was also plotted in Figure 8(a). As a result, the multi-QTF based "on-beam" spectrophone showed the signal amplitude of $58.8 \mathrm{mV}$, yielding a 1.4 times enhancement compared to the traditional single QTF based "on-beam" spectrophone and a 33 times enhancement compared to the single bare QTF based spectrophone. The asymmetry of the signal was due to the residual amplitude modulation of the laser [15].

The performance of the multi-QTF based "on-beam" spectrophone in Figure 4(d) was evaluated in the same way. The comparisons in the resonance curves and the signal amplitudes were shown in Table 2 and Figure 8(b), respectively. In contrast with the spectrophone in Figure 4(c), the 


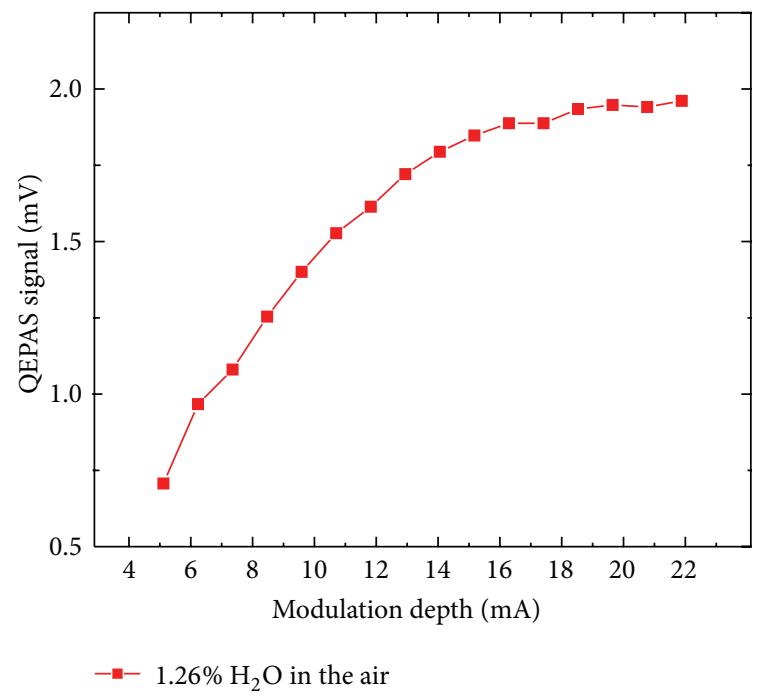

(a)

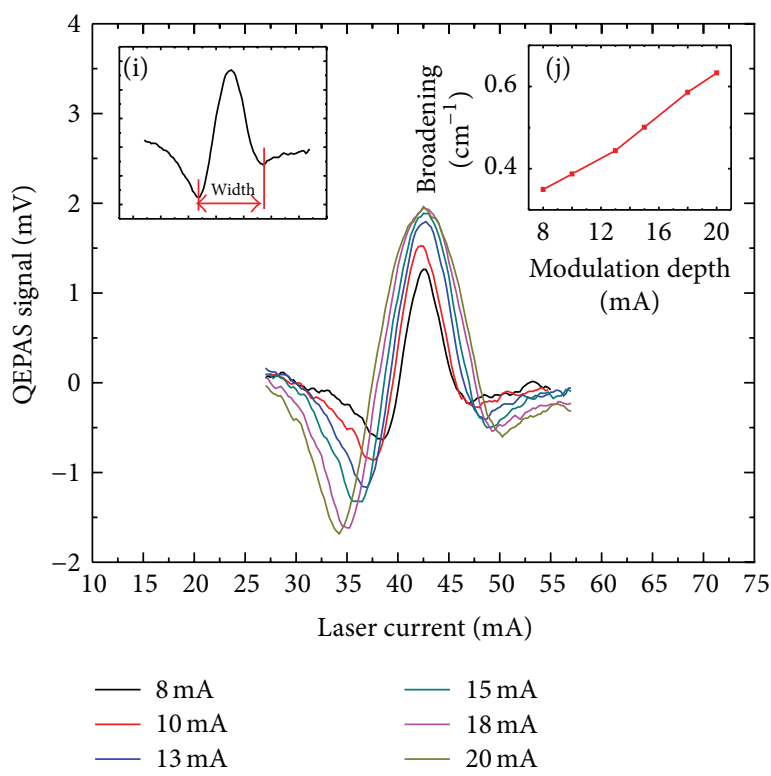

(b)

FIGURE 7: (a) QEPAS signal amplitudes as a function of laser modulation depth; (b) spectral broadening widths of QEPAS signal as a function of laser modulation depth.

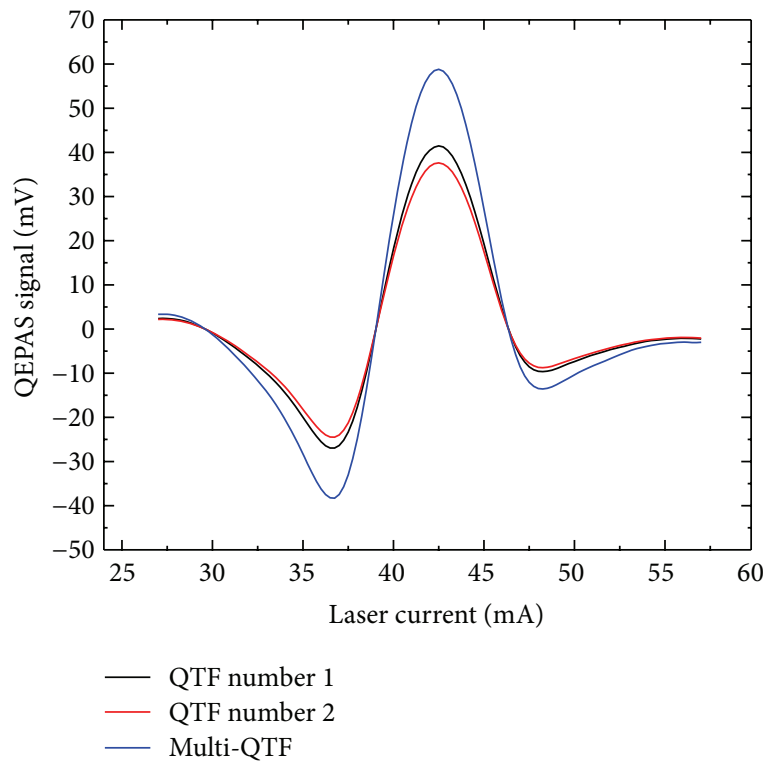

(a)

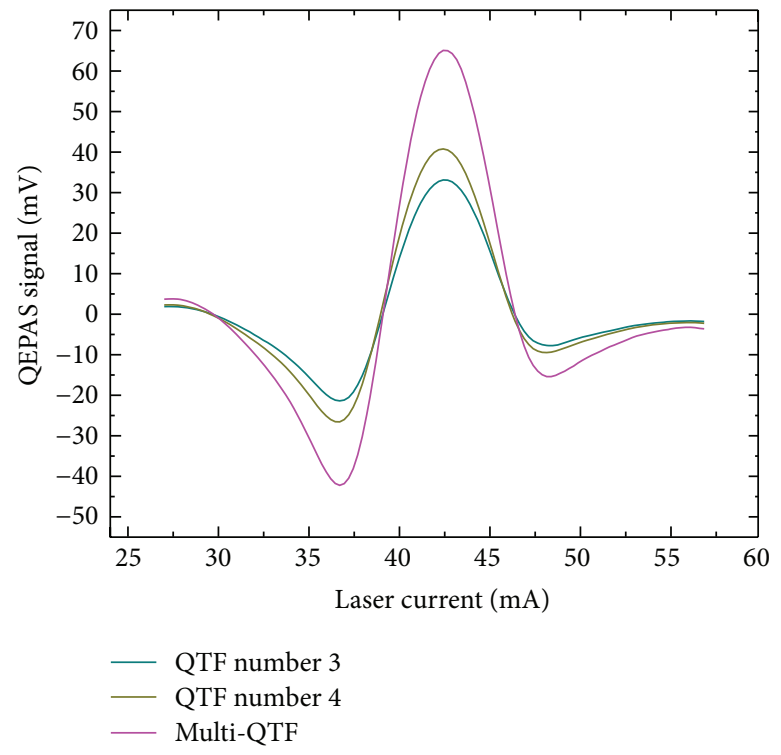

(b)

Figure 8: (a) Performance of multi-QTF based "on-beam" spectrophone shown in Figure 4(c); (b) performance of multi-QTF based "onbeam" spectrophone shown in Figure 4(d).

multi-QTF based "on-beam" spectrophone in Figure 4(d) generated a signal amplitude of $65.1 \mathrm{mV}$ which is 1.6 times higher than that of the traditional single QTF based "onbeam" spectrophone and 36 times higher than that of the single bare QTF based spectrophone. Considering the higher signal gain, the multi-QTF based "on-beam" spectrophone in Figure $4(\mathrm{~d})$ is preferred.

The QEPAS signal amplitude of the multi-QTF based "onbeam" spectrophone (Figure $4(\mathrm{~d})$ ) as a function of distance between QTF \#4 and AmR \#4 was optimized and shown in Figure 9. With the distance increasing from 10 to $50 \mu \mathrm{m}$, the signal amplitudes achieve the maximum value of $65.1 \mathrm{mV}$ at the distance of $40 \mu \mathrm{m}$, as shown in the inset of Figure 9 . This observed behavior is due to the coupling effect of the acoustic wave fields between QTF \#4 and the AmRs. The signal-to-noise ratio (SNR) of the sensor can be calculated from the $2 f$ signal maximum value and the noise background level obtained by tuning the laser wavelength far away from 


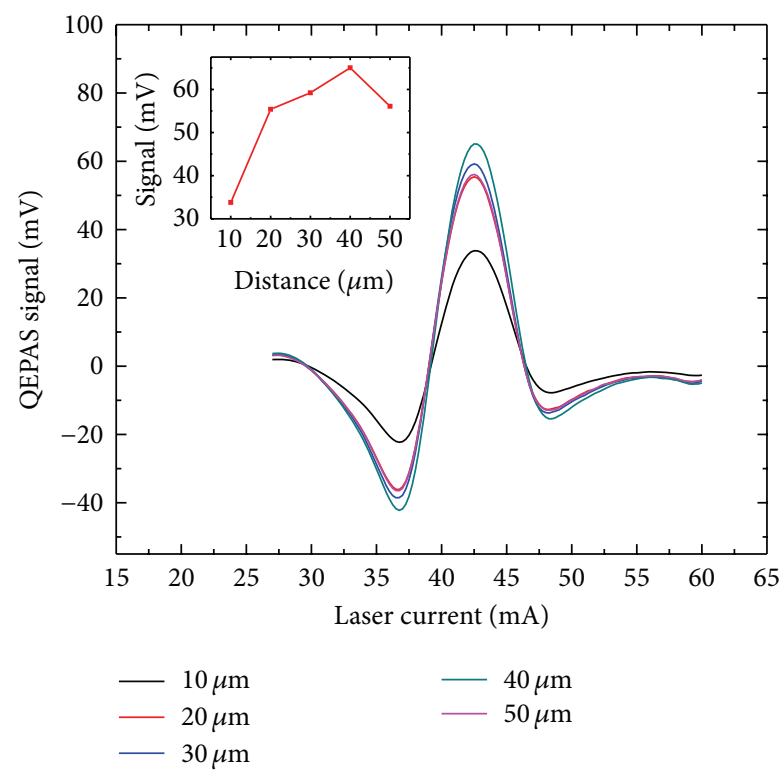

FIGURE 9: Signal amplitudes of the multi-QTF based "on-beam" spectrophone (Figure $4(\mathrm{~d})$ ) as a function of distance between QTF \#4 and AmR \#4.

the $\mathrm{H}_{2} \mathrm{O}$ absorption lines. With the $1 \sigma$ noise of $4.6 \times 10^{-5} \mathrm{~V}$, the SNR of the multi-QTF based "on-beam" spectrophone (Figure 4(d)) is 1410, corresponding to a detection limit of $9.96 \mathrm{ppm}$. Normalized to the excitation power of $5.4 \mathrm{~mW}$ and the detection bandwidth of $0.125 \mathrm{~Hz}$, the obtained $1 \sigma$ normalized noise equivalent absorption (NNEA) coefficients were $1.24 \times 10^{-9} \mathrm{~W} \cdot \mathrm{cm}^{-1} \cdot \mathrm{Hz}^{-1 / 2}$.

\section{Conclusions}

Acoustic microresonators are added to the most recently developed multi-QTF based QEPAS sensor to further enhance the signal amplitude. Two kinds of multi-QTF based spectrophones with "on-beam" AmR configurations were experimentally investigated and discussed. Compared with the traditional single QTF based "on-beam" spectrophone, the developed multi-QTF based "on-beam" spectrophone had a signal enhancement of 1.6 times at the same operating conditions, with the approximate noise level. A normalized equivalent absorption coefficient $(1 \sigma)$ of $1.24 \times$ $10^{-9} \mathrm{~W} \cdot \mathrm{cm}^{-1} \cdot \mathrm{Hz}^{-1 / 2}$ was obtained for water vapor detection at normal atmospheric pressure. The signal enhancement can be further improved by using a phase shifter to adjust the possible phase difference between the multi-QTFs.

\section{Conflict of Interests}

The authors declare that there is no conflict of interests regarding the publication of this paper.

\section{Acknowledgments}

The work was supported by the 973 program (Grant no. 2012CB921603), the National Natural Science Foundation of
China (Grant nos. 61575113, 61275213, 61108030, 61475093, 61127017, 61178009, 61378047, and 61205216), the National Key Technology R\&D Program (2013BAC14B01), the Shanxi Natural Science Foundation (2013021004-1), and the Shanxi Scholarship Council of China (2013-011, 2013-01).

\section{References}

[1] L. Dong, H. Wu, H. Zheng et al., "Double acoustic microresonator quartz-enhanced photoacoustic spectroscopy," Optics Letters, vol. 39, no. 8, pp. 2479-2482, 2014.

[2] H. Wu, L. Dong, H. Zheng et al., "Enhanced near-infrared QEPAS sensor for sub-ppm level $\mathrm{H}_{2} \mathrm{~S}$ detection by means of a fiber amplified $1582 \mathrm{~nm}$ DFB laser," Sensors and Actuators B: Chemical, vol. 221, pp. 666-672, 2015.

[3] H. Yi, R. Maamary, X. Gao, M. W. Sigrist, E. Fertein, and W. Chen, "Short-lived species detection of nitrous acid by external-cavity quantum cascade laser based quartz-enhanced photoacoustic absorption spectroscopy," Applied Physics Letters, vol. 106, no. 10, Article ID 101109, 2015.

[4] A. Rosencwaig, Photoacoustics and Photoacoustic Spectroscopy, Wiley, 1980.

[5] P. Patimisco, G. Scamarcio, F. K. Tittel, and V. Spagnolo, "Quartz-enhanced photoacoustic spectroscopy: a review," Sensors, vol. 14, no. 4, pp. 6165-6206, 2014.

[6] H. Wu, L. Dong, W. Ren et al., "Position effects of acoustic micro-resonator in quartz enhanced photoacoustic spectroscopy," Sensors and Actuators B: Chemical, vol. 206, pp. 364-370, 2014.

[7] H. Wu, A. Sampaolo, L. Dong et al., "Quartz enhanced photoacoustic $\mathrm{H}_{2} \mathrm{~S}$ gas sensor based on a fiber-amplifier source and a custom tuning fork with large prong spacing," Applied Physics Letters, vol. 107, no. 11, Article ID 111104, 2015.

[8] Y. Ma, G. Yu, J. Zhang, X. Yu, R. Sun, and F. K. Tittel, "Quartz enhanced photoacoustic spectroscopy based trace gas sensors using different quartz tuning forks," Sensors, vol. 15, no. 4, pp. 7596-7604, 2015.

[9] L. Dong, A. A. Kosterev, D. Thomazy, and F. K. Tittel, "QEPAS spectrophones: design, optimization, and performance," Applied Physics B: Lasers and Optics, vol. 100, no. 3, pp. 627-635, 2010.

[10] K. Liu, X. Guo, H. Yi, W. Chen, W. Zhang, and X. Gao, "Offbeam quartz-enhanced photoacoustic spectroscopy," Optics Letters, vol. 34, no. 10, pp. 1594-1596, 2009.

[11] Y. Ma, X. Yu, G. Yu et al., "Multi-quartz-enhanced photoacoustic spectroscopy," Applied Physics Letters, vol. 107, no. 2, Article ID 021106, 2015.

[12] H. Yi, W. Chen, X. Guo et al., "An acoustic model for microresonator in on-beam quartz-enhanced photoacoustic spectroscopy," Applied Physics B, vol. 108, no. 2, pp. 361-367, 2012.

[13] H. Zheng, L. Dong, X. Yin et al., "Ppb-level QEPAS $\mathrm{NO}_{2}$ sensor by use of electrical modulation cancellation method with a high power blue LED," Sensors and Actuators, B: Chemical, vol. 208, pp. 173-179, 2015.

[14] A. A. Kosterev, F. K. Tittel, D. V. Serebryakov, A. L. Malinovsky, and I. V. Morozov, "Applications of quartz tuning forks in spectroscopic gas sensing," Review of Scientific Instruments, vol. 76, no. 4, Article ID 043105, 2005.

[15] Y. Cao, W. Jin, H. L. Ho, L. Qi, and Y. H. Yang, "Acetylene detection based on diode laser QEPAS: combined wavelength and residual amplitude modulation," Applied Physics B, vol. 109, no. 2, pp. 359-366, 2012. 

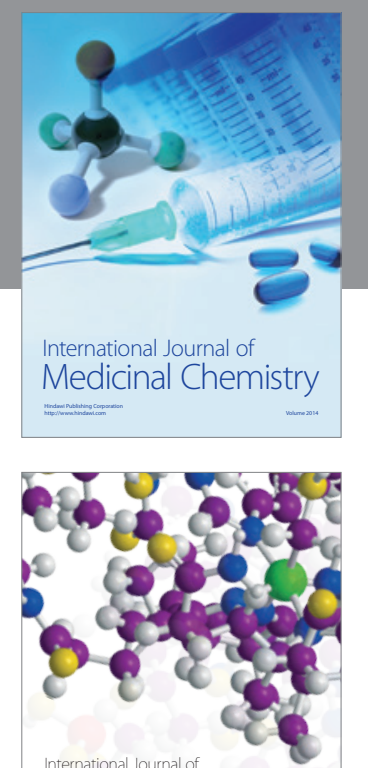

\section{Carbohydrate} Chemistry

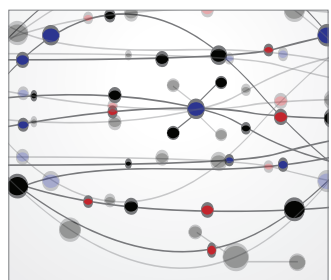

The Scientific World Journal
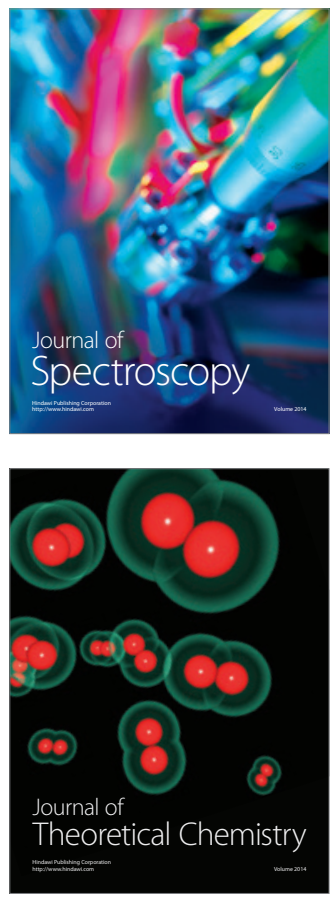
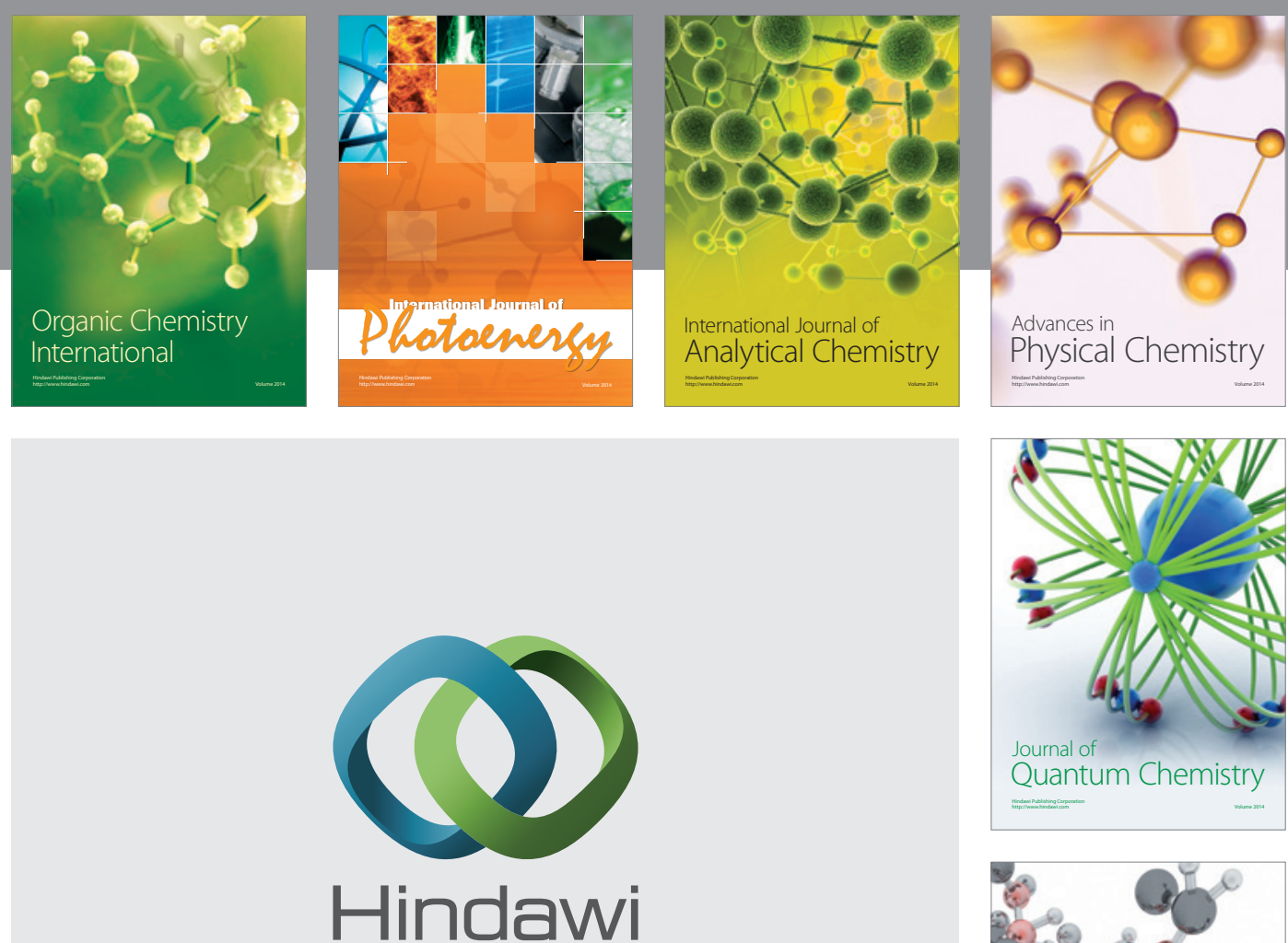

Submit your manuscripts at

http://www.hindawi.com

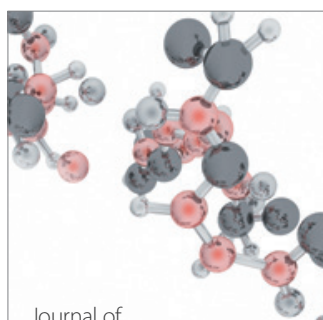

Analytical Methods

in Chemistry

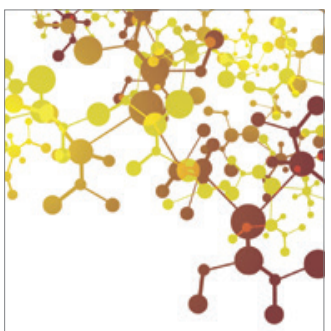

Journal of

Applied Chemistry

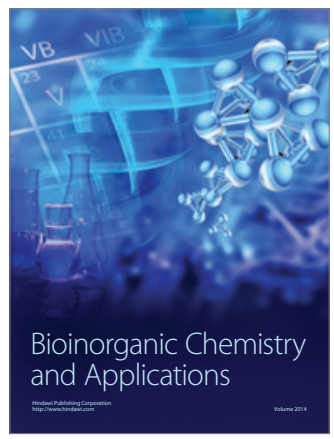

Inorganic Chemistry
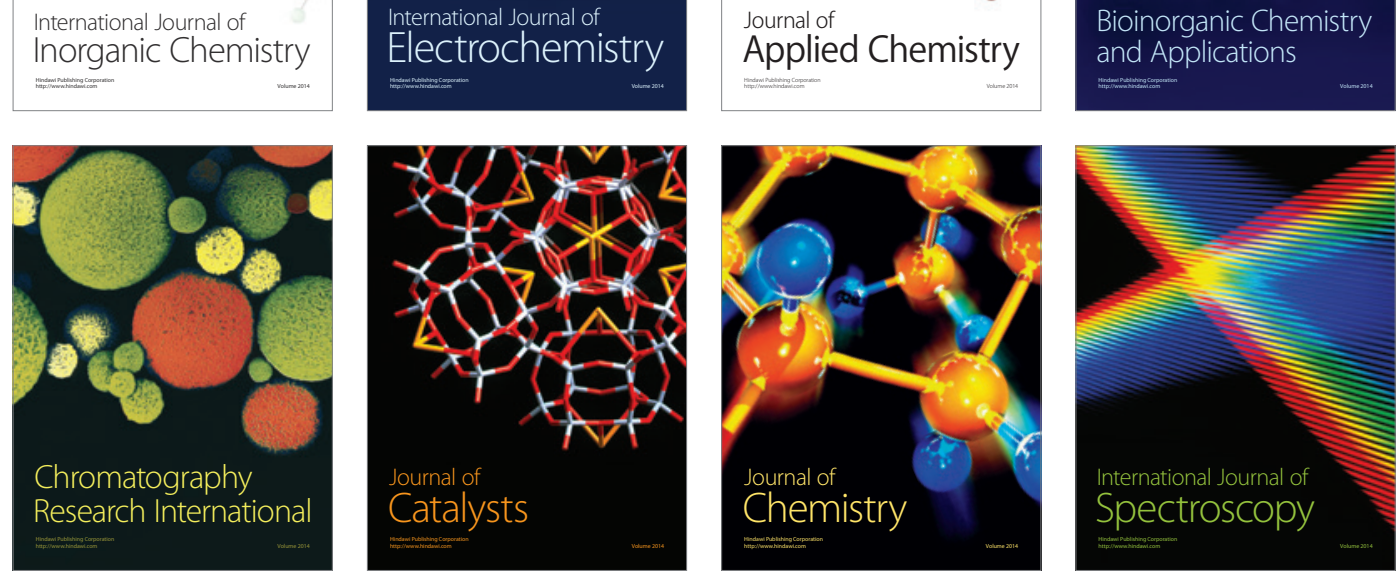